\title{
Impacto de la globalización en el tráfico de mujeres y niñas con fines de explotación sexual
}

\author{
Alexa Corena' \\ Universidad Autónoma de Colombia
}

Artículo de Reflexión derivado de investigación

Recibido: abril 10 de 2015 - Aprobado: mayo 20 de 2015

\section{Resumen}

La globalización como fenómeno económico ha beneficiado el comercio del sexo, siendo éste un mercado transnacional cuyo supuesto subyace en el pretendido de que los dueños del capital y de las mercancías buscan las condiciones más rentables para reproducirlo, ya que el trabajo de esclavas sexuales viabiliza la obtención de una altísima utilidad en la medida en la que el "empresario" se siente desobligado a pagar a las "trabajadoras", que son de su propiedad una remuneración por su labor, pues el negocio lleva implícita la condición del pago de deudas adquiridas por las mujeres y niñas que no han sido concertadas previamente en una relación comercial equitativa, sino en una situación forzosa y engañosa.

Desde este enfoque, el presente documento pretende mostrar cómo la trata de mujeres y niñas con fines de explotación sexual se potencia en el entorno globalizado dadas las facilidades que surgen en las fronteras difusas entre países y en los marcos jurídicos locales que se mezclan con el entorno internacional para favorecer mafias transnacionales que, en el amparo de actividades legales (como el modelaje, las niñeras, el turismo, etc.), captura mujeres y niñas, que son trasladadas a países diferentes en donde son indocumentadas, aisladas y vulneradas.

Palabras Clave: Globalización, tráfico de personas, derechos humanos, nuevo orden mundial, mafias transnacionales, cultura patriarcal, explotación sexual de mujeres, violencia hacia mujeres.

I Economista de la Universidad Autónoma de Colombia. Ms. En Educación del Instituto Tecnológico de Monterrey. Ms. En Género, Sociedad y Políticas de FLACSO en trabajo de Investigación. Jefe del Área de Promoción Socioeconómica. Universidad Autónoma de Colombia. Investigadora y Líder del Grupo de Investigación Modus Vivendi, adscrito a la Línea de investigación: Comunidad Universitaria. Dirección electrónica:alcorena@gmail.com 


\title{
Impact of globalization on women and girls trafficking aimed at sexual exploitation
}

\begin{abstract}
Globalization as an economic phenomenon has benefitted sex trade, which is a transnational market based on the idea that the owners of the capital and merchandises look for the most profitable conditions to reproduce it. Sexual slave work makes it easy to obtain a very high return, as the "manager" does not feel any obligation to pay "workers" any money as they belong to him/her given that the business implies that women and girls are to pay their debts, which were not previously agreed on in an egalitarian business relationship, but acquired in a forced deceitful way.

From this approach, the present paper intends to show how girls and women trafficking with the purpose of sexual exploitation is enhanced in the globalized environment given the easiness that emerges within the diffuse boundaries among countries and local legal frameworks which mix with international ones to favor transnational mafias that, under the shelter of legal activities like modeling, au pairing, tourism, etc., capture women and girls and transfer them to countries where they become illegal, isolated and deprived.
\end{abstract}

Key words: globalization, people trafficking, human rights, new global order, transnational mafias, patriarchal culture, sexual exploitation of women, violence against women

\section{Resumo}

\section{Impacto da globalização no tráfico de mulheres e meninas com fins de exploração sexual}

A globalização como fenômeno econômico tem beneficiado o comércio do sexo, sendo este um mercado transnacional, cujo suposto sobejasse na pretensão de que os donos do capital e das mercadorias buscam as condições mais rentáveis para reproduzi-lo, já que o trabalho de escravas sexuais viabiliza a obtenção de uma altíssima utilidade na medida em que o "empresário" se sente desobrigado a pagar às "trabalhadoras" que são de sua propriedade uma remuneração pelo seu lavor, pois o negócio leva implícita a condição do pagamento de dívidas adquiridas pelas mulheres e as meninas que não tem sido concertadas previamente numa relação comercial equitativa, mas numa situação forçosa e enganosa.

Partindo deste enfoque, o presente documento pretende mostrar como a trata de mulheres e meninas com fins de exploração sexual se potencia no entrono globalizado, dadas as facilidades que surgem nas fronteiras difusas entre países e nos marcos jurídicos locais que se misturam com o entorno internacional para favorecer máfias transnacionais que, no amparo de atividades legais (como as modelos, as babás e o turismo), captura mulheres e meninas que são trasladadas a países diferentes onde são indocumentadas, isoladas e vulneradas.

Palavras chave: Globalização, tráfico de pessoas, direitos humanos, nova ordem mundial, máfias transnacionais, cultural patriarcal, exploração sexual de mulheres, violência contra as mulheres. 


\section{Introducción}

El 23 de septiembre de cada año, se conmemora en el mundo el Día Internacional contra la Explotación Sexual y el Tráfico de Mujeres, Niñas y Niños, el cual fue instaurado por la Conferencia Mundial de la Coalición Contra el Tráfico de Personas a partir de la Conferencia de Mujeres que tuvo lugar en Dhaka, Bangladesh, en enero de 1999, siendo importante recordar, de acuerdo a la formulación que presenta la $\mathrm{ONU}$, que la trata de personas se caracteriza por "la acción de captar, transportar, trasladar, acoger o recibir personas, recurriendo a la amenaza o al uso de la fuerza u otras formas de coacción, al rapto, al fraude, al engaño, al abuso de poder o de una situación de vulnerabilidad o a la concesión o recepción de pagos o beneficios para obtener el consentimiento de una persona que tenga autoridad sobre otra con fines de explotación" ?

En el mismo sentido y bajo un concepto más amplio, la OIT³ , trabaja por conformar una alianza global, que oriente sus esfuerzos en la consolidación de un marco legal y en los procesos de sensibilización social frente al problema, siendo importante resaltar la definición que hace esta organización del trabajo forzado referenciándolo como la explotación que se ejerce sobre las personas que son obligadas a realizar un trabajo en condiciones de sometimiento. Esto, teniendo en cuenta que el entorno globalizado favorece la trata de mujeres y niñas con fines de explotación sexual a partir de la posición de esta actividad en el mercado, así como por las múltiples interrelaciones que surgen entre agentes mundiales que trafican personas como si fuesen mercancías, que maximizan la utilidad de mercancías que se transan en las fronteras difusas entre países, bajo lógicas de mercado y amparándose en vacíos profundos existentes en los marcos jurídicos que favorecen mafias transnacionales, las cuales, a su vez, aprecian la soberanía de los estados como un poder borroso y de alcance limitado, es decir, una "soberanía dividida y maniatada" 4.

El presente artículo además, aborda el tema de las causas originarias y perpetuadoras del tráfico de mujeres y niñas para actividades de prostitución, no solamente como consecuencia de la cultura patriarcal, sino además desde la óptica de la violencia contra las mujeres y niñas suscitada por el Estado que excluye a la población más vulnerable al asumir un papel pasivo en la protección de sus derechos económicos y sociales, concretamente al desentenderse de su obligación de garantizarles acceso a educación, salud, vivienda, trabajo digno, etc. Adicionalmente porque las entidades que deben velar por la aplicación de la justicia, y en este marco de acción investigar, juzgar y sancionar los

2 Naciones Unidas. Protocolo para prevenir, reprimir y sancionar la trata de personas, especialmente mujeres y niños, que complementa la Convención de las Naciones contra la Delincuencia Organizada Transnacional publicado en Convención de las Naciones Unidas contra la delincuencia organizada Transnacional y sus protocolos. Nueva York. 2004

3 Organización Internacional del Trabajo (OIT), (2005). Una alianza global contra el trabajo forzoso. Informe global con arreglo al seguimiento de la Declaración de la OIT relativa a los principios y derechos fundamentales en el trabajo. Conferencia Internacional del Trabajo. 93a reunión, 2005. Ginebra.

4 McGrew, A y Held, D. (2003). Globalización/Antiglobalización. Sobre la reconstrucción del orden mundial. Colección Estado y Sociedad, Paidós, Oxford. 
crímenes cometidos, no desarrollan sus funciones ni actúan con criterios de verdad y ética, lo cual genera alta impunidad, que incrementan los índices de violencia y criminalidad ${ }^{5}$.

\section{Marco Contextual. El nuevo orden mundial}

De acuerdo a Todaro ${ }^{6}$, la globalización, la internacionalización y la multinacionalización son tres fenómenos distintos, siendo el primero una de las etapas del proceso evolutivo del sistema económico mundial, que se caracteriza por lograr que ciertas actividades, inicialmente locales, logren funcionar, en tiempo real, como una sola a nivel universal ${ }^{7}$.

De allí que Sklair ${ }^{8}$, evidencie en el proceso globalizador la creación, mantenimiento y reproducción de múltiples vínculos e interconexiones que surgen en los mercados y se extienden más allá de los límites territoriales y simbólicos de los países, lo cual es elemento fundamental de la nueva configuración mundial que se caracteriza, según McGrew, A ${ }^{9}$ por la interdependencia de las relaciones mundiales representadas en acaecimientos, disposiciones y prácticas que afectan a personas y comunidades del mundo, indistintamente de sus orígenes e historias.

En este contexto se genera un nuevo orden mundial que permea todas las esferas de los países y de las personas, generando resultados significativos y diferentes, caracterizado por un proceso permanente de creación de riqueza desigual y articulada a las condiciones iniciales de unos y otros, es decir, se crea riqueza y pobreza. Adicionalmente, surge un conjunto de actores influyentes que operan en una lógica independiente a la de los países del mundo: las empresas transnacionales, que operan a nivel internacional y logran que los capitales nacionales se integren a ellas y a esa lógica mundial impuesta por ellas ${ }^{10}$, siendo fundamental resaltar que al interior de esta gran alianza confluyen ejecutivos, burócratas, políticos y gobernantes que actúan como transmisores ideológicos que traspasan sus beneficios e inciden en los lobbies políticos para lograr sus resultados $"$.

Así las cosas, las corporaciones transnacionales, los medios de comunicación y los movimientos sociales brotan como actores fundamentales de un gran modelo de sistema capitalista mundial, citado

5 Zúñiga, A. E. (2014). Derechos Económicos- Sociales y Violencia Facilitador de Acciones Estratégicas en Honduras. CIPREVI. Centro de Investigación para la prevención de la violencia. Honduras. Documento disponible en: http://ciprevica.org/download/ reflexiones_semanales/DDHH-Violencia_HN.pdf

6 Todaro, R. (2000). Aspectos de género de la globalización y la pobreza. Documento disponible en: http://www.un.org/womenwatch/ daw/csw/todaro.htm

7 Castells, (1999). Globalización, Identidad y Estado en América Latina, Ministerio Secretaría General de la Presidencia -Chile- y PNUD. Santiago

8 Sklair, L. (20 I0) PRIGGEP. Maestría en Género, Sociedad y Políticas. Aula virtual. Información encontrada en: http://wmw.prigepp. org/aula/unidad_20 I 0.php?CodigoUni=\&CodigoCap $=139 \&$ CodigoSca=240\&CodigoCop=324\&CodigoPub=\&imprime= |

9 McGrew, A y Held, D. Op. Cit.

10 Todaro. Op. Cit.

I I Sklair. Op. Cit. 
por Sklair ${ }^{12}$, el cual está conformado por una multiplicidad de interacciones entre agentes gubernamentales y no gubernamentales que operan atravesando las fronteras de los países, edificándose un plano de los "poderosos del mundo" que corporizan su praxis en las instituciones, liderando de esta manera prácticas económicas.

Con la globalización surgen nuevos espacios, mercados y actividades integradas que implican operaciones que apoyan a la gran empresa global, lo cual ha implicado nuevas condiciones para la mano de obra, la cultura laboral y otros elementos que antes eran de resorte de las economías locales, pero que ahora requieren homogenizarse para estar al servicio de este nuevo orden mundial ${ }^{13}$, dando como resultado la transformación del sistema mundial de producción, creándose nuevas formas de organización entre las empresas, interconectado los mercados financieros, los flujos de capital y en general lográndose un ajuste estructural que implica la creación de marcos regulatorios nacionales e internacionales requeridos para la globalización y, por supuesto para la ampliación de la economía de mercado que finalmente, ha conseguido el efecto de la desigualdad profunda ${ }^{14}$.

Para la adaptación de las economías locales al contexto mundial, se han introducido desde fuera de sí mismas, los siguientes cambios estructurales, citados por Todaro 15: a) reducciones a la carga impositiva que grava las utilidades de las empresas por la vía de las reformas tributarias, b) empequeñecimiento del estado de bienestar dado por la reducción del gasto público para lo cual se han realizado importantes reducciones del gasto en salud, educación, vivienda y programas de apoyo social, c) disminución de las costos laborales a las empresas por las nuevas legislaciones laborales que implican precarización del empleo formal d) eliminación o minimización de políticas de pleno empleo, aumentándose el riesgo de pérdida del empleo, de las prestaciones de seguridad social y estabilidad laboral, e) transformación de los sistemas de seguridad social basados en el reparto solidario hacia sistemas basados en el individuo con el consiguiente aumento de la vulnerabilidad personal).

Las citadas transformaciones están orientadas a lograr la competitividad de las empresas, sin que sea importante que se generen disparidades regionales, desigualdades entre sectores socioeconómicos y grupos de personas, muchos de los cuales han visto deteriorada su calidad de vida. De esta manera y progresivamente, se viven en el mundo momentos de recesión y pobreza para amplios sectores de la población y un aumento de las brechas entre ricos y pobres, así como en el acceso a recursos, muchos de ellos vitales y generadores de progreso.

Dentro del descrito escenario de mercado que favorece a las empresas transnacionales, la globalización es factor que ha patrocinado el comercio del sexo, llevándolo al estatus de mercado trasnacional bajo supuestos del libre mercado, defensa de la propiedad del capital y de las mercancías, siendo éste un escenario en el que se encuentran las condiciones más rentables para la utilidad, de

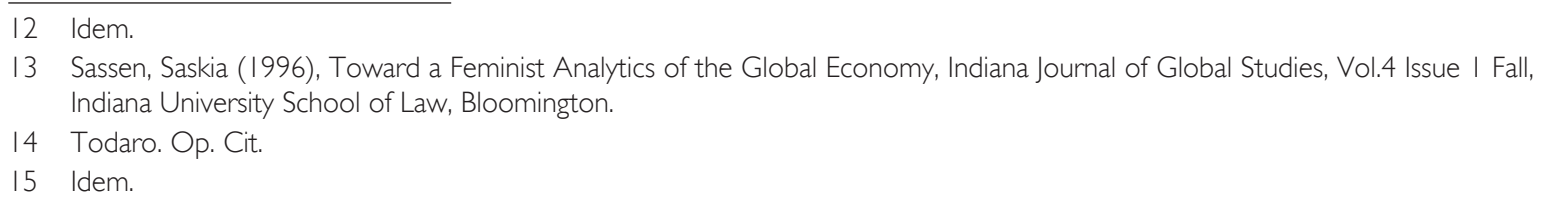


allí que el trabajo de esclavas sexuales produzca altas utilidades, pues se basa en la no obligatoriedad de remunerar a las "trabajadoras".

En la misma línea de pensamiento Sklair ${ }^{16}$, sostiene que las "ideologías del consumismo" y del desarrollo sustentable se han constituido en ejes concluyentes de las prácticas simbólicas que involucran la globalización y que llevan a que se produzca una voracidad excesiva de las corporaciones que se centran en acopiar beneficios excesivos a costa de la fuerza de trabajo, los consumidores y las sociedades locales.

De allí que es pertinente retomar las afirmaciones de Roberto Toscano, quien plantea que en el escenario mundial se promueve una "globalización de aspiraciones" por alcanzar ciertos estándares de consumo y estilos de vida, pero no se ocupa de una "globalización de la oportunidad económica". En este sentido, el autor muestra cómo prevalecen las leyes de la competencia por encima de las instituciones y de los derechos de las personas, siendo prioritarios para garantizarles una vida más humana, especialmente para todos aquellos individuos menos poderosos ${ }^{17}$.

Así, es pertinente remitirse al planteamiento de Sklair ${ }^{18}$, en relación con la composición del actual modelo de sistema capitalista global, el cual, de acuerdo con el autor, está conformado por diversos agentes de la sociedad que interactúan, incluso más allá de la fronteras del Estado, generándose una red que construye prácticas transnacionales que se enquistan en la misma estructura, permeando las instituciones y reproduciéndose reproduce a través de estos agentes estatales y no estatales que son sus perpetuadores.

Bajo tales planteamientos, Sklair ${ }^{19}$, muestra la conformación de elites poderosas mundialmente, lideradas por trasnacionales que lideran las prácticas económicas, en las cuales actores tales como ejecutivos, burócratas, políticos y profesionales globalizados, se convierten en sus trasmisores ideológicos en la medida en que inciden en los escenarios políticos para lograr su aceptación y legitimación.

En el mismo sentido, explican Gios, L. Vena, M. y Marambio M.A. ${ }^{20}$, en este contexto particular, estos actores estructuran redes de crimen organizado, con alcance nacional e internacional que operan por la división estructurada del trabajo, la planificación de la actividad delictiva, la corrupción de agentes del Estado en todos los niveles, el beneplácito de la estructura social, la legalización de las actividades en la estructura económica, la vulneración de la identidad y la comunicación de las mujeres traficadas, al ser incomunicadas e indocumentadas en países en donde son extranjeras; la restricción de la libertad personal, las sanciones y acciones disciplinarias.

16 Sklair. Op. Cit.

17 Toscano, R. (200 I). Interrogantes éticos sobre la globalización. Publicado en: Estado constitucional y Globalización. Universidad Nacional Autónoma de México. Editorial Porrúa. México.

18 Sklair. Op. Cit.

19 Idem.

20 Gios, L. Vena, M. y Marambio M.A. (20 I3). La Trata De Personas Con Fines de explotación sexual como una nueva forma de esclavitud. Universidad Nacional del Centro de la Provincia de Buenos Aires. UNICEN. Documento disponible en: http://www.unicen.edu. ar/content/la-trata-de-personas-con-fines-de-explotaci\%C3\%B3n-sexual-como-una-nueva-forma-de-esclavitud 


\section{El tráfico de personas, actividad violatoria de los derechos humanos}

De acuerdo con lo publicado en Ajuntament de Dénia ${ }^{21}$, el tráfico de personas, en este caso mujeres y niñas, para la explotación sexual es un delito que afecta derechos humanos fundamentales tales como el derecho a la vida, a la integridad física, psíquica, a la seguridad, a la libertad y a la dignidad de la persona. De la misma manera, vulnera también otra serie de derechos humanos entre los cuales se encuentran el derecho a no ser sometida a esclavitud, a no ser vendida en matrimonio, torturada, sometida a tratos inhumanos, crueles o degradantes y con un mayor alcance, el derecho a una vida familiar, a la intimidad, a la salud, a una vivienda en condiciones de seguridad y a no ser discriminada por el hecho de ser mujer.

Es de considerar el argumento de la OIT 22, al señalar que la explotación económica forzosa de personas en el mundo ocurre en sectores económicos a los que acude una gran masa poblacional con menores calificaciones y alta vulnerabilidad, tal es el caso de los ámbitos relacionados con la agricultura, la construcción, la fabricación de ladrillos, los talleres manufactureros informales, afectándose, en proporciones similares a hombres y mujeres, siendo diferente para la explotación forzosa sexual con propósitos comerciales que afecta principalmente a mujeres y a niñas ${ }^{23}$.

Desde el punto de vista del género, plantea Todaro ${ }^{24}$, en el contexto globalizado las mujeres se presentan como un grupo poblacional altamente vulnerable a la precarización laboral, debido a que están propensas a aceptar trabajos de peor calidad que les permita cumplir su rol familiar, lo cual implica relaciones laborales inestables, desprotección laboral y social; mayor posibilidad de estar excluidas de procesos de capacitación y formación indispensables para la inserción laboral, asegurándose de esta manera una mano de obra barata, necesaria para el funcionamiento de la economía global.

Es de anotar que en el escenario referido, según la propuesta de Büchner ${ }^{25}$,la globalización está marginando a los más desfavorecidos, es decir, mujeres, pobres y culturas no occidentales, agravando sus condiciones y generándose una feminización de la pobreza, lo cual se evidencia, tal como plantea la autora en que el $70 \%$ de los pobres sean mujeres. De la misma manera, de acuerdo con el Informe sobre Desarrollo Humano de 1995 "las mujeres realizan más de la mitad del trabajo en el mundo y, sin embargo, sólo un tercio del mismo es remunerado", lo cual implica un mayor desempleo femenino basado en la cultura patriarcal que plantea las tareas del hogar de exclusivo desempeño de la mujer, lo cual conlleva a menor poder adquisitivo y de decisión en las mujeres más vulnerables, lo cual es apoyado por las mafias transnacionales que encuentran en este sustrato la oportunidad para esclavizar a partir del planteamiento de promesas de bienestar y futuro deseable, mujeres que viven y se mantienen en la miseria.

21 Ajuntament de Dénia, (20I0). 23 de septiembre, Día Internacional contra la explotación sexual y la trata de personas. Artículo disponible en: http://www.denia.es/es/informacio/actualitat/new.aspx?id=1798

22 OIT. Op. Cit.

23 Idem.

24 Todaro. Op. Cit.

25 Büchner, J. (2004). Género y Globalización. Versión reducida de la ponencia presentada en el Grupo de Estudios Críticos. Documento disponible en: http://www.mujeresenred.net/spip.php?article844 


\section{Factores que inciden}

Tal como lo señala Audet ${ }^{26}$, la explotación sexual de mujeres y niñas persiste y cobra mayor relevancia gracias a diferentes factores, entre los cuales es fundamental reconocer que desde la cultura patriarcal se legitima el uso de las mujeres y niñas en actividades de prostitución dado que aún se les ve como objetos de consumo. De esta manera, plantea la autora, se valida la violencia hacia mujeres y niñas a partir de que es normal que los hombres necesiten satisfacerse sexualmente, en todo momento y en cualquier parte, por lo que se justifica que paguen por comprar el cuerpo de una mujer, o una niña/o.

Este argumento coincide con lo expuesto por Pineda ${ }^{27}$, quien señala el hecho de que históricamente la mujer ha sido considerada objeto que sacia los apetitos sexuales del hombre y posibilita el ejercicio del poder masculino, por lo que, plantea la autora, se entiende que la mujer debe cumplir con este papel voluntariamente o no, esto teniendo en cuenta que en la lógica androcéntrica y misógina, no existe autonomía de las mujeres sobre su cuerpo, construyéndose desde estos imaginarios el complejo sistema de explotación a la mujer, justificándose además la comercialización, Pineda ${ }^{28}$

En este escenario el mismo autor llama la atención en el encubrimiento de cualquier delito que pueda surgir en el mercado del sexo en la medida en la que se juzga y se señala a las mujeres prostitutas, pero no en la misma forma a las mafias y a los proxenetas que conforman redes delictivas y mucho menos a quienes compran estos servicios, es decir a los clientes, cuando estos últimos son el eje del negocio.

De otra parte, de acuerdo con Méndez ${ }^{29}$, los medios de comunicación masivos juegan un papel fundamental en la medida en la que promueven todo tipo de consumo, sin que se excluya lo relacionado con los servicios sexuales, para lo cual trabajan con pautas que se enfocan en incrementar el erotismo hacia las mujeres, y de la misma manera, reproduciendo las bases de la cultura patriarcal, se les presenta como objetos sexuales, capaces de satisfacer cualquier requerimiento masculino, fortaleciendo el constructo socio-cultural de dominación del hombre sobre la mujer, otorgando un marco simbólico a la prostitución, la trata de mujeres y niñas, el secuestro, la explotación y la esclavitud.

Desde el punto de vista de los derechos económicos y sociales, Zúñiga, A. E. ${ }^{30}$, plantea la responsabilidad del Estado que excluye a la población más vulnerable al asumir un papel pasivo en la protección de sus derechos económicos y sociales, concretamente al desentenderse de su obligación

26 Audet, E. (2003). Prostitución, tráfico sexual y globalización. Agencia Latinoamericana de Información ALAl, Publicado en América Latina en Movimiento, No. 370: http://alainet.org/publica/370.phtmlérica Latina en Movimiento.2003-06-10

27 Pineda E. (2012). Globalización y esclavitud sexual. Documento disponible en: www.aporrea.org/actualidad/a l 39203.html

28 Idem.

29 Méndez, S. (201 I). Explotación sexual. El tráfico de mujeres y niñas. Comisión Provincial por la Memoria - CPM. Publicado en el Tiempo Argentino. Articulo disponible en: http://tiempo.infonews.com/notas/trafico-de-mujeres-y-nina

30 Zúñiga, A. E. Op. Cit. 
de garantizarles acceso a educación, salud, vivienda, trabajo digno, etc. Adicionalmente porque las entidades que deben velar por la aplicación de la justicia, y en este marco de acción investigar, juzgar y sancionar los crímenes cometidos, no desarrollan sus funciones ni actúan con criterios de verdad y ética, lo cual genera alta impunidad, que incrementa los índices de violencia y criminalidad, lo cual se ve agravado cuando se suma la penetración de agentes de las mafias en la estructura de justicia de cada país, volviendo más complejo el entorno y profundizando aún más la impunidad que nace en las entrañas del Estado mismo, así como la credibilidad de la población en el mismo.

Según Gios, L. Vena, M. y Marambio M.A. ${ }^{31}$, aun los gobiernos latinoamericanos no reconocen abiertamente el delito de trata de mujeres y niñas para la explotación sexual, por lo que los marcos normativos y las estructuras judiciales, no han incorporado legislaciones específicas que den importancia a la gravedad del delito e involucre tantas acciones como mecanismos y estrategias sean necesarias para detectar el problema, así como para acompañar e intervenir poblaciones en riesgo y víctimas, y por supuesto para investigar jurídicamente y procesar a los traficantes. De la misma manera, las autoras consideran fundamental generar lazos de cooperación entre los diferentes cuerpos de seguridad que puedan tener contacto directo o indirecto tanto con víctimas como con victimarios.

\section{La trata de blancas en el mundo globalizado}

El tráfico de mujeres y niñas para la explotación sexual se establece como un negocio nacional, internacional y transnacional, que se constituye en la tercer actividad ilegal que mayores dividendos genera en el mundo, logrando registrar ventas anuales de 7.000 millones de dólares, siendo en realidad un hecho de esclavitud y violación de derechos humanos, lo cual es referenciado por Gios, L. Vena, M. y Marambio M.A. ${ }^{32}$.

En el mismo sentido, Audet, E. ${ }^{33}$, señala que esta industria, según cifras de las Naciones Unidas generó en el año 2000, alrededor de 8 a 9 millones de euros, además presenta cifras de la Interpol que muestran cómo una sola prostituta entrega a su proxeneta un promedio de 108.000 euros anuales. De allí que la autora manifieste que esta actividad genera más dividendos a las mafias que la operan que las obtenidas por el negocio del tráfico de armas o de drogas, esto debido a que una sola mujer o niña que es comercializada muchas veces genera un efecto multiplicador de la utilidad.

En este contexto, Geadah, Y. ${ }^{34}$, presenta una variante del negocio transnacional que es el turismo sexual, escenario que no se trabaja con la exportación de mujeres y niñas, sino con la atracción de consumidores a destinos turísticos, generalmente ubicados en países pobres, y lo presenta como una

31 Gios, L. Vena, M. y Marambio M.A. Op. cit.

32 Idem.

33 Audet. Op. Cit.

34 Geadah, Y. (2003), La prostitution un métier comme un autre?, Montréal, VLB, 2003. Síntesis y traducción libre del artículo producido en Sysphie, mayo 2003, Quebec. Publicado en América Latina en Movimiento, No. 370: http://alainet.org/publica/370.phtml 
variante el tráfico sexual transnacional anotando que, en este escenario no se trata de "exportar" mujeres y niñas/os, sino de atraer consumidores hacia los países pobres.

En términos globales Marina, J.A y De la Valgoma, $M^{35}$, evidencian cómo las industrias del sexo no solamente crecen y se reproducen, sino además son motores de desarrollo de las nuevas tecnología. Y no es para menos si se considera la importancia económica de un negocio que mueve más de 3.100 millones de pesetas anuales a través de internet.

\section{Conclusiones}

Después de analizar el fenómeno desde diferentes aristas, se concluye en consonancia con lo expuesto por Marina, J.A y De la Valgoma, M. ${ }^{36}$,que en el entorno global, la trata de mujeres y niñas cumple con todas las características del mercado ya que existe oferta, demanda, mercancía, utilidad maximizada a bajos costos, satisfacción de las necesidades del cliente, generación de riqueza y marcos legales que legitiman la actividad, todo independiente de que la mercancía transada sean seres vivos a quienes se les están vulnerando sus derechos.

Desde el punto de vista de las soluciones, de acuerdo con los planteamientos del Instituto del Tercer Mundo ${ }^{37}$, es necesario que los países desarrollen marcos normativos y políticas públicas orientadas a penalizar el delito incluyendo todos y cada uno de los actores que participan, además a trabajar en la reducción de la pobreza. En el mismo sentido, a ubicar el fenómeno en el contexto globalizado para lograr la cooperación internacional en la abolición de la trata de personas y de la esclavitud, para lo cual, de acuerdo a Audet, es fundamental considerar todas y cada una de las dimensiones del problema, es decir las humanas, sociales, políticas y éticas.

Como parte integral del marco normativo y de las políticas públicas, se requiere que los estados trabajen en prevenir, investigar, y reparar a las mujeres, niñas, niños víctimas de la trata con fines de explotación sexual, además de repararles otorgándoles tal estatus, lo cual significa que se les posibilite y garantice atención psico-sanitaria, reinserción socio-económica y oportunidades laborales dignas.

Bajo dicha propuesta es necesario que los gobiernos nacionales dediquen un gran esfuerzo en la inversión pública para garantizar a las poblaciones excluidas sus derechos económicos y sociales, teniendo en cuenta que esta desigualdad es el sustrato en el cual surgen y se reproducen acciones delictivas que, como el tráfico de mujeres y niñas para la explotación sexual, se surten de personas que sueñan con un mejor futuro y huyen de la pobreza y la violencia.

De la misma manera, en relación con la propuesta de Nielen Héller, los países deben trabajar

35 Marina, J.A y De la Valgoma, M (200 I), Esclavitud y globalización. Publicado en el diario EL MUNDO el 10 de junio de 200 I.

36 Idem.

37 Instituto del Tercer Mundo (autoría; canal) (2005). Autores corporativos. Esclavitud y globalización: variables y réditos. Fecha: 22 de Mayo de 2005 
en la edificación de estructuras jurídicas, políticas y sociales robustas que den cuenta de un Estado garante de la protección de los sujetos frente al riesgo económico y social, así como preservar los derechos humanos y la ciudadanía, avanzando en doble vía: de una parte sancionando a aquellos actores que de manera activa participan en la actividad delictiva de comercio de mujeres y niñas con cualquier fin ilegal; de otra parte con el diseño e implementación de políticas públicas de prevención, contención y reinserción de quienes han caído en estas situaciones y requieren de un apoyo estatal decidido para evitar que caigan de nuevo en las situaciones de explotación y esclavitud sexual ${ }^{38}$.

\section{Referencias}

Ajuntament de Dénia, (2010). 23 de septiembre, Día Internacional contra la explotación sexual y la trata de personas. Artículo disponible en: http://www.denia.es/es/informacio/actualitat/new.aspx?id=1798

Audet, E. (2003). Prostitución, tráfico sexual y globalización. Agencia Latinoamericana de Información ALAl, Publicado en América Latina en Movimiento, No. 370: http://alainet.org/publica/370.phtmlérica Latina en Movimiento.2003-06-10

Baumann Z. (1999). Turistas y vagabundos. La globalización: consecuencias humanas. FCE, Buenos Aires, Documento trabajado en la Unidad No. 2. Seminario PRIGEPP-FLACSO.

Büchner, J. (2004). Género y Globalización. Versión reducida de la ponencia presentada en el Grupo de Estudios Críticos. Documento disponible en: http://www.mujeresenred.net/spip.php?article844

Castells, (1999). Globalización, Identidad y Estado en América Latina, Ministerio Secretaría General de la Presidencia -Chile- y PNUD. Santiago

Geadah, Y. (2003), La prostitution un métier comme un autre?, Montréal, VLB, 2003. Síntesis y traducción libre del artículo producido en Sysphie, mayo 2003, Quebec. Publicado en América Latina en Movimiento, No. 370: http://alainet.org/publica/370.phtml

Gios, L. Vena, M. y Marambio M.A. (20 I 3). La Trata De Personas Con Fines de explotación sexual como una nueva forma de esclavitud. Universidad Nacional del Centro de la Provincia de Buenos Aires. UNICEN. Documento disponible en: http://www.unicen.edu.ar/content/la-trata-de-personas-con-fines-de-explotaci\%C3\%B3n-sexualcomo-una-nueva-forma-de-esclavitud

Instituto del Tercer Mundo (autoría; canal) (2005). Autores corporativos. Esclavitud y globalización: variables y réditos. Fecha: 22 de Mayo de 2005

McGrew, A y Held, D. (2003). Globalización/Antiglobalización. Sobre la reconstrucción del orden mundial. Colección Estado y Sociedad. Oxford. Paidós.

38 Sklair. Op. Cit. 
Marina, J.A y De la Valgoma, M (200 I), Esclavitud y globalización. Publicado en el diario EL MUNDO el 10 de junio de 2001 .

Méndez, S. (20I I). Explotación sexual. El tráfico de mujeres y niñas. Comisión Provincial por la Memoria - CPM. Publicado en el Tiempo Argentino. Articulo disponible en: http://tiempo.infonews.com/notas/trafico-demujeres-y-nina

Organización Internacional del Trabajo (OIT), (2005). Una alianza global contra el trabajo forzoso. Informe global con arreglo al seguimiento de la Declaración de la OIT relativa a los principios y derechos fundamentales en el trabajo. Conferencia Internacional del Trabajo. 93a reunión, 2005. Ginebra.

Pineda E. (20|2). Globalización y esclavitud sexual. Documento disponible en: www.aporrea.org/actualidad/ al 39203.html

Sklair, L. (20 I0) PRIGGEP. Maestría en Género, Sociedad y Políticas. Aula virtual. Información encontrada en:

http://www.prigepp.org/aula/unidad_20 10.php?CodigoUni=\&CodigoCap=139\&CodigoSca=240\&CodigoCop=3 24\&CodigoPub=\&imprime $=1$

. Sassen, Saskia (1998), Globalization and Its Discontents. The New Press, New York.

Toscano, R. (200 I). Interrogantes éticos sobre la globalización. Publicado en: Estado constitucional y Globalización. Universidad Nacional Autónoma de México. Editorial Porrua. México.

Todaro, R. (2000). Aspectos de género de la globalización y la pobreza. Documento disponible en: http://www. un.org/womenwatch/daw/csw/todaro.htm

Zúñiga, A. E. (2014). Derechos Económicos- Sociales y Violencia Facilitador de Acciones Estratégicas en Honduras. CIPREVI. Centro de Investigación para la prevención de la violencia. Honduras. Documento disponible en: http://ciprevica.org/download/reflexiones_semanales/DDHH-Violencia_HN.pdf 